\title{
Correspondence
}

Korean J Ophthalmol 2021;35(5):410-412 https://doi.org/10.3341/kjo.2021.0046

\section{Inferior Rectus Hypoplasia Simulat- ing Contralateral Hypotropia: A Pediatric Case Report}

\section{Dear Editor,}

This report presents a case of inferior rectus muscle hypoplasia, which was treated successfully through a recession procedure with posterior fixation of the contralateral inferior rectus. A healthy 4-month-old male infant with left eye hypotropia appeared 1 month after birth visited a strabismus clinic. The patient was delivered normally, with no sign of infection. Previous medical and family histories were insignificant. The performance in fix and follow of an object $15 \mathrm{~cm}$ from the face was normal in the right eye but showed moderate impairment in the left eye. The cycloplegic refraction results were +1.00 diopter sphere, -1.00 diopter cylinder $\times 180^{\circ}$, and +0.75 and $-0.50 \times 180^{\circ}$ in the right and left eyes, respectively. The Krimsky test revealed 25 prism diopters (PD) of left eye hypotropia and 8 PD exophoria in primary gaze. Ductions and versions revealed one degree of supraduction deficit in the left eye, while infraduction could not be evaluated due to the patient's inability to cooperate. Ptosis, abnormal head posture, and facial asymmetry were not present. T2-weighted coronal and sagittal imaging of the orbit and high-resolution magnetic resonance imaging of the brain stem evaluated extraocular muscles and cranial nerves, respectively. The high-resolution magnetic resonance imaging revealed hypoplasia of the inferior rectus muscle in the right eye, which was half the diameter of the left eye inferior rectus muscle (Fig. 1A$1 \mathrm{~F})$. The other extraocular muscles appeared normal in

Received: March 31, 2021 Final revision: June 25, 2021

Accepted: July 15, 2021 both eyes. The bilateral oculomotor nerve appeared to be normal.

The patient was diagnosed with congenital right eye inferior rectus hypoplasia. Occlusion therapy was performed on the left eye amblyopia, but the performance in fix and follow had not improved. Therefore, $5.0-\mathrm{mm}$ right eye superior rectus recession and 5.0-mm left eye inferior rectus recession with posterior fixation were performed. At 2 years after surgery, the performance in fix and follow of the left eye was normal. Right eye hypertropia was $<5$ PD for both far and near vision.

Idiopathic congenital inferior rectus hypoplasia is a rare disease with only one previously reported case in South Korea [1]. Embryologically, the inferior rectus, inferior oblique, and lateral rectus muscles originate from the inferior mesodermal complex [1]. Any problem affecting inferior mesodermal complex development can induce medical or radiologic abnormalities, such as in the present case. The bilateral oculomotor nerves were normal and symmetrical in our patient. This indicates that inferior rectus hypoplasia development has a different pathogenesis than congenital cranial disintegration disorders such as Duane retraction syndrome, congenital fibrosis syndrome, and congenital oculomotor nerve palsy [2]. We assume that inferior rectus muscle hypoplasia is caused by abnormalities of the oculomotor nerve fascicles [3].

The manifestations of congenital inferior rectus hypoplasia or aplasia are as follows: increased hypertropia during abduction, decreased hypertropia and limitation of infraduction during ipsilateral head tilting, A-pattern strabismus, diplopia exacerbation during infraduction, chindown posture, and intorsion. Inferior rectus aplasia or hypoplasia, and not inferior rectus muscle palsy, are considered when the above clinical signs are shown [4].

In children who are unable to cooperate with the strabismus examination, inferior rectus hypoplasia can be misdiagnosed as monocular elevation deficiency. If dominant 

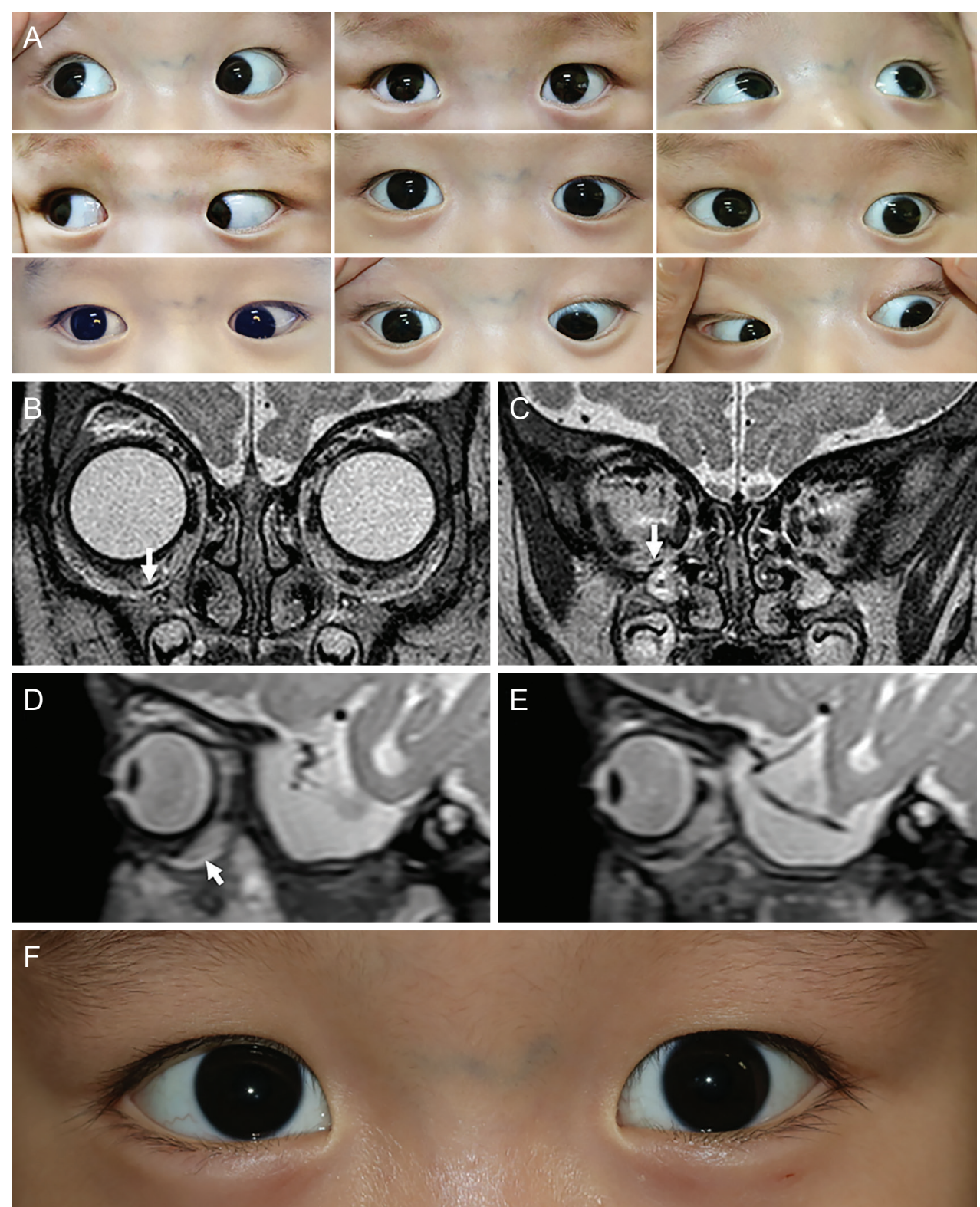

Fig. 1. Right inferior rectus hypoplasia. (A) Gaze photographs demonstrating left hypotropia and limited infraduction in the right eye. (B-E) Coronal and sagittal T2-weighted orbital high-resolution magnetic resonance imaging shows hypoplastic inferior rectus muscle in right eye. The muscle diameter of the right inferior rectus muscle was the half diameter of the left inferior rectus. (B-E) Arrows indicate hypoplastic right inferior rectus muscle. (F) The primary gaze photo of 2 years after the surgery. Informed consent for publication of the clinical images was obtained from the parents of the patient.

eye of patients with inferior rectus hypoplasia is the contralateral eye, definite supraduction limitation can appear in the contralateral eye through increased signals of secondary deviation [3]. In this case, covering the affected eye and examining movement of the contralateral eye are important for preventing misdiagnosis. High-resolution radiologic tests are also essential for diagnosing extraocular hypoplasia.

Posterior fixation procedure is a muscle weakening technique that does not disturb the primary gaze balance. The indications for posterior fixation are dissociated vertical deviation, double-elevator paresis, Duane retraction syndrome, and incomitant strabismus [5]. Based on Hering's law, posterior fixation sutures can increase the stimulation 
of the yoke muscle due to decreased torsional force on the affected eye. In the present case, the permanent infraduction limitation was estimated, and posterior fixation suture was delivered to the unaffected eye [2].

A case of idiopathic congenital inferior rectus hypoplasia has been identified, which as disguised as hypotropia and supraduction limitation in the contralateral eye. Inhibitional palsy can cause the misdiagnosis of inferior rectus hypoplasia as contralateral elevation deficiency. Thorough duction and version tests and radiologic examinations are essential for diagnosing inferior rectus hypoplasia. Posterior fixation sutures combined with recession of the contralateral inferior rectus muscle can correct vertical deviation due to unilateral inferior rectus hypoplasia.

Yeon Jeong Lee, Yung Ju Yoo

Department of Ophthalmology, Kangwon National University School of Medicine, Chuncheon, Korea

E-mail (YungJu Yoo): yyjuuuu@kangwon.ac.kr

\section{Conflict of Interest}

No potential conflict of interest relevant to this article was reported.

\section{Acknowledgements}

This article was supported by 2021 Kangwon National University Hospital.

\section{References}

1. Kim E, Kim JH, Hwang JM, et al. MR imaging of congenital or developmental neuropathic strabismus: common and uncommon findings. AJNR Am J Neuroradiol 2012;33:205661.

2. Engle EC, Goumnerov BC, McKeown CA, et al. Oculomotor nerve and muscle abnormalities in congenital fibrosis of the extraocular muscles. Ann Neurol 1997;41:314-25.

3. Kim JM, Kim JH, Hwang JM. Inferior rectus aplasia simulating contralateral monocular elevation deficiency. $J$ Pediatr Ophthalmol Strabismus 2010;47 Online:e1-3.

4. Fan YY, Yang ML. Managing hypoplasia of the inferior rectus muscle by inferior oblique anterior transposition in children. J Pediatr Ophthalmol Strabismus 2017;54:e50-3.

5. von Noorden GK. Inidcations of the posterior fixation operation in strabismus. Ophthalmology 1978;85:512-20. 